\title{
Measured distortion of a laminar circular Couette flow by end effects
}

\author{
By D. COLES AND C. VAN ATTA $†$ \\ Graduate Aeronautical Laboratories, California Institute of Technology
}

(Received 2 August 1965)

Measurements at moderately large Reynolds numbers in a finite laminar circular Couette flow show that the tangential motion near the axial plane of symmetry is two-dimensional, within experimental accuracy, but is nevertheless strongly modified by end conditions.

During the course of an experimental investigation of transition in circular Couette flow (Van Atta 1966) it became necessary to measure very accurately the laminar velocity profile midway between the ends of a finite rotating-cylinder apparatus. The formal object of the measurements was to establish a known flow for calibration of a hot-wire probe array for response to changes in pitch angle, yaw angle, and speed. The simplest laminar motion which could conveniently be set up in the apparatus at hand was a flow with the inner cylinder at rest and with the outer cylinder and end plates rotating together. The measurements were made at relatively high laminar Reynolds numbers, and they showed the influence of the end plates on the tangential flow field to be unexpectedly large. The experiment is therefore reported here for its intrinsic interest as an example of a three-dimensional secondary flow in a rotating fluid.

The experimental technique was to carry out a simultaneous flow survey and probe calibration by holding the cylinder speed constant and traversing a hot-wire probe radially in the initially unknown flow. Electrical signals from the probe were then extrapolated to the far wall, where the fluid velocity with respect to the probe was accurately known. Several such extrapolations at different fixed cylinder speeds provided a relationship between velocity and voltage for use away from the wall. To keep the relative velocity at the probe as large as possible, the outer half of the flow was surveyed from the stationary inner cylinder, and the inner half of the flow was surveyed from the rotating outer cylinder. In both cases, however, the wires were supplied with current through slip rings. The same hot wires, slip rings, cables, and instrumentation were used through the measurements, which extended over a period of several weeks.

The platinum-rhodium hot wires used had a diameter of 0.0001 in. and a length of about $0.04 \mathrm{in}$, and were operated in the constant-temperature mode (hot resistance/cold resistance about 1.15). All wires were mounted parallel to the axis of rotation of the cylinders. Two wires on a single probe were traversed 
radially, while a third wire was fixed at mid-radius $180^{\circ}$ away on the same cylinder to observe the wake of the movable probe.

During a typical traverse, the outer cylinder was run at constant speed, and the data probe was retracted in several steps from an initial position near the far wall to a final position near mid-radius. At the two extreme probe positions the outer cylinder was stopped, and the distance from the data wires to the opposite wall was measured optically using a cathetometer. With the outer cylinder again running, the probe was then returned in several steps to its original position. Intermediate radial positions were measured without stopping the apparatus by using an electronic counter to count revolutions of a small servo motor driving the data probe in the radial direction. Any discrepancy between the radial probe displacement as measured optically and as inferred from the total counter reading was blamed on counts missed during the starting and stopping of the traverse motor, and was distributed equally among the several increments of radial motion. The final values for probe position are believed to be accurate to $\pm 0.002 \mathrm{in}$. or better. The radius of the outer cylinder was $17.998 \mathrm{in}$. (nominally $18 \mathrm{in}$.), and the radius of the inner cylinder was $16.001 \mathrm{in.} \mathrm{(nominally} 16 \mathrm{in}$.). The distance between the inside surfaces of the transparent end closures was $54.7 \mathrm{in}$.

The period of rotation of the outer cylinder was measured using pulses from a magnetic pickup to start and stop an electronic counter. The speed regulation over long periods of time was about one part in $10^{3}$. The room temperature (about $23^{\circ} \mathrm{C}$ ) was taken as the average reading of two mercury thermometers at the two ends of the apparatus. The wire cold resistance was checked once a day using a bridge circuit. The constant hot resistance was inferred from one set of measurements of current and voltage. Atmospheric pressure (about $29.3 \mathrm{in} . \mathrm{Hg}$ ) was read with a mercury barometer.

Voltages were measured using a voltage-to-frequency converter and an electronic counter. The resolution of the combination was about one part in $10^{4}$ $(0.0001 \mathrm{~V}$ in $1.4 \mathrm{~V})$. A zener-stabilized reference voltage source, which had previously been checked against a primary standard, was read at frequent intervals to disclose any drift in the digital voltmeter.

In a view looking along the horizontal axis of rotation, the outer cylinder and the end plates rotated clockwise, and the optical station was at 9 o'clock. At the optical station, therefore, a probe on the inner cylinder pointed down, and a probe on the outer cylinder pointed up. During each optical measurement of probe position, the free-convection heat loss was also measured. Although this loss depended slightly on radial probe position when the probe was less than $0.5 \mathrm{in}$. from the wall, the heat-loss data at larger distances provided an accurate measure of drift in the hot-wire voltage under nominally fixed conditions. This drift was probably caused by temperature dependence of base-emitter current in a balanced transistor amplifier circuit. Interpolation between measured values of freeconvection heat loss ensured that suitably normalized data for inward and outward traverses would agree, other things being equal.

From previous work with similar hot-wire instrumentation in the same apparatus, it was known that the data could be adequately fitted in the range of 
measurement by the usual form of King's law for Nusselt number as a function of the Reynolds number,

$$
N u=A+B \sqrt{ } R e,
$$

where $A$ and $B$ are empirical constants. By definition,

$$
N u=\frac{i^{2} R_{h} / \pi l d}{k\left(T_{h}-T_{c}\right) / d}, \quad R e=\frac{\rho v d}{\mu},
$$

with $i$ the wire current $R_{h}$ the wire hot resistance, $l$ the wire length, $d$ the wire diameter, $T_{h}$ the wire hot temperature, $T_{c}$ the air temperature, $k$ the air heat conductivity, $\rho$ the air density, $v$ the relative velocity, and $\mu$ the air viscosity. It was also known that the air properties $\rho, k$, and $\mu$ could be evaluated at the (constant) wire hot temperature $T_{h}$. Several of the variables in $N u$ and $R e$ could therefore be suppressed in the data reduction, leaving King's law in the simplified form

$$
n / n_{0}=\alpha+\beta \sqrt{ } v
$$

where $\alpha$ and $\beta$ are now empirical constants (neglecting a slight dependence of $\beta$ on pressure), and

$$
n=e^{2} /\left(R_{h}-R_{c}\right),
$$

with $e$ the wire voltage and $R_{c}$ the wire resistance at room temperature. The free-convection heat loss $n_{0}$ (interpolated between measured values) was included to minimize the effect of drift in the hot-wire amplifiers. The cold resistance $R_{c}$ at any given air temperature was inferred using the known temperature coefficient of resistance for the wire. Inasmuch as both the hot and cold wire resistances included the same small contributions from cables and slip rings, the differences $R_{h}-R_{c}$ were accurately determined. The actual wire voltages were not, but there is no question that the operating conditions for the wires were precisely defined.

The crucial operation of extrapolation of the probe data to the wall was carried out in two steps. In the first step, $n / n_{0}$ was plotted against radius and extrapolated to the wall more or less by eye, without much regard for the analytical form of the profiles. The object was to obtain a first approximation for the intercept $\alpha$ in the simplified version of King's law by plotting $\left(n / n_{0}\right)_{w}$ against $\sqrt{ } v_{w}$, the subscript $w$ denoting a value at the wall. Given this estimate for $\alpha$, the second step was to plot $\left(n / n_{0}-\alpha\right)^{2}$ against radius. This second quantity, being essentially proportional to the velocity, could be extrapolated more accurately by using the method illustrated in figure 1 . It was assumed that the velocity profile near either wall could be adequately fitted by the sum of a constant term, a term in $y$, and a term in $y^{2}$, with $y$ the distance from the wall. By adding a quadratic term to $\left(n / n_{0}-\alpha\right)^{2}$, such that the sum became essentially a straight line, and by extrapolating this line to the wall, a second and final relationship between $\left(n / n_{0}\right)_{w}$ and $\sqrt{ } v_{w}$ was obtained. This final relationship, as illustrated by some typical data in figure 2, was then used to determine the velocity throughout the flow.

The resulting velocity data, averaged for the two moving hot wires, are shown in figure 3. Except for a handful of points, the two velocities always agreed to within a fraction of $1 \%$. Some of the measurements were also repeated with the 


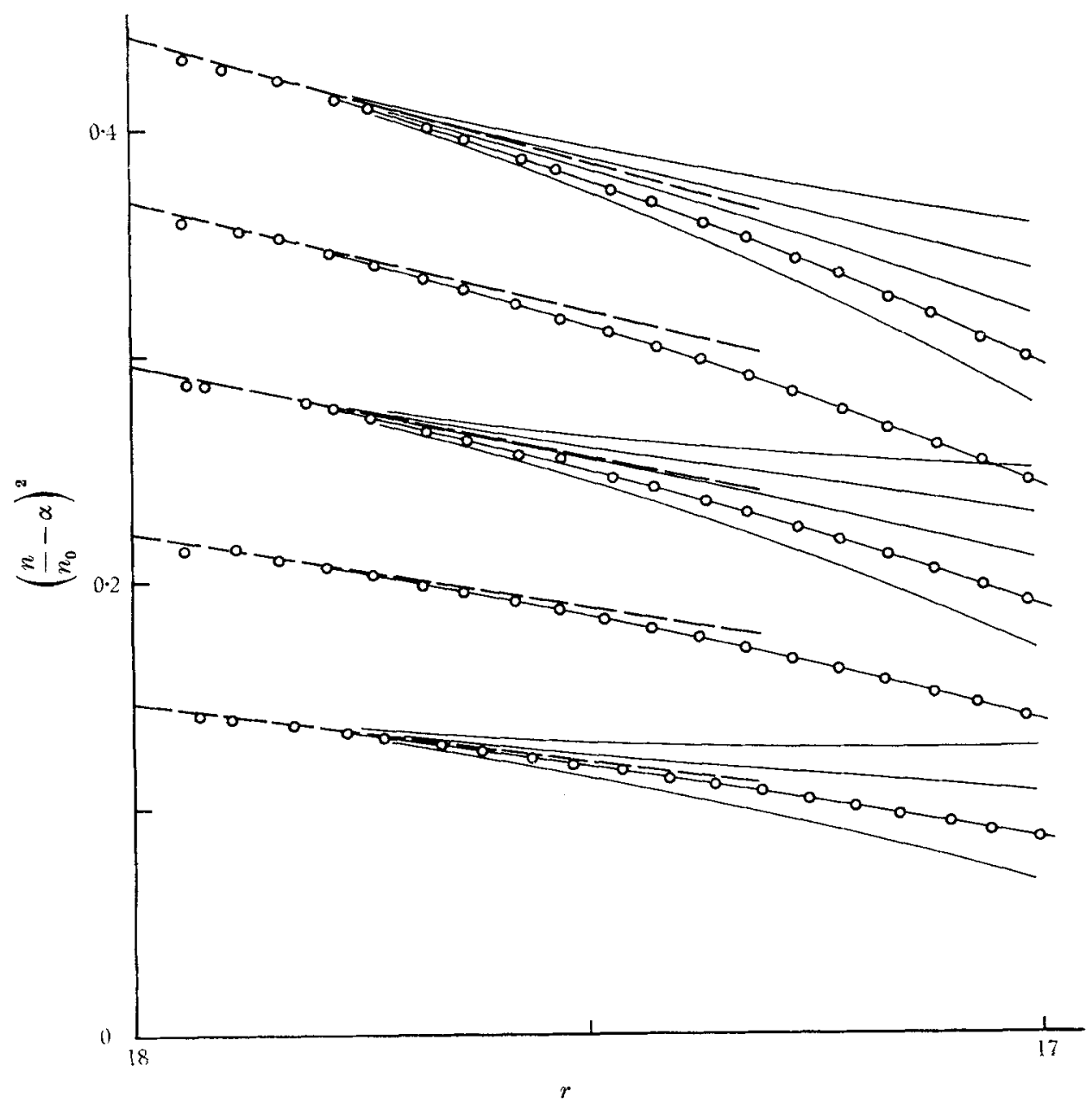

Figure $1(a)$. For legend see facing page.

data probe pitched $\pm 10^{\circ}$ or yawed $\pm 15^{\circ}$. No effect of probe orientation was found. Interference by the wall, however, could be detected at appreciable distances. At the lower end of the speed range the measurements had to be cut off at a relative velocity of about $1.5 \mathrm{ft}$./ $/ \mathrm{sec}$, because heat loss by free convection became noticeable, even at the low overheat ratio used (cf. figure 2). The measurements were also limited to relative velocities less than about $9 \mathrm{ft}$./sec, because of instability of the probe wake. This instability tripped the flow; i.e. caused it to change spontaneously to an alternative intermittently turbulent flow, for probe Reynolds numbers (based on stem diameter and tip relative velocity) greater than about 300 . Points where this type of transition occurred, as the probe was extended or the speed was increased, are marked by stars in figure 3. Once turbulence had appeared in the bi-stable flow as a result of the finite disturbance from the probe, this turbulence would persist until the speed was decreased to quite a low value. 


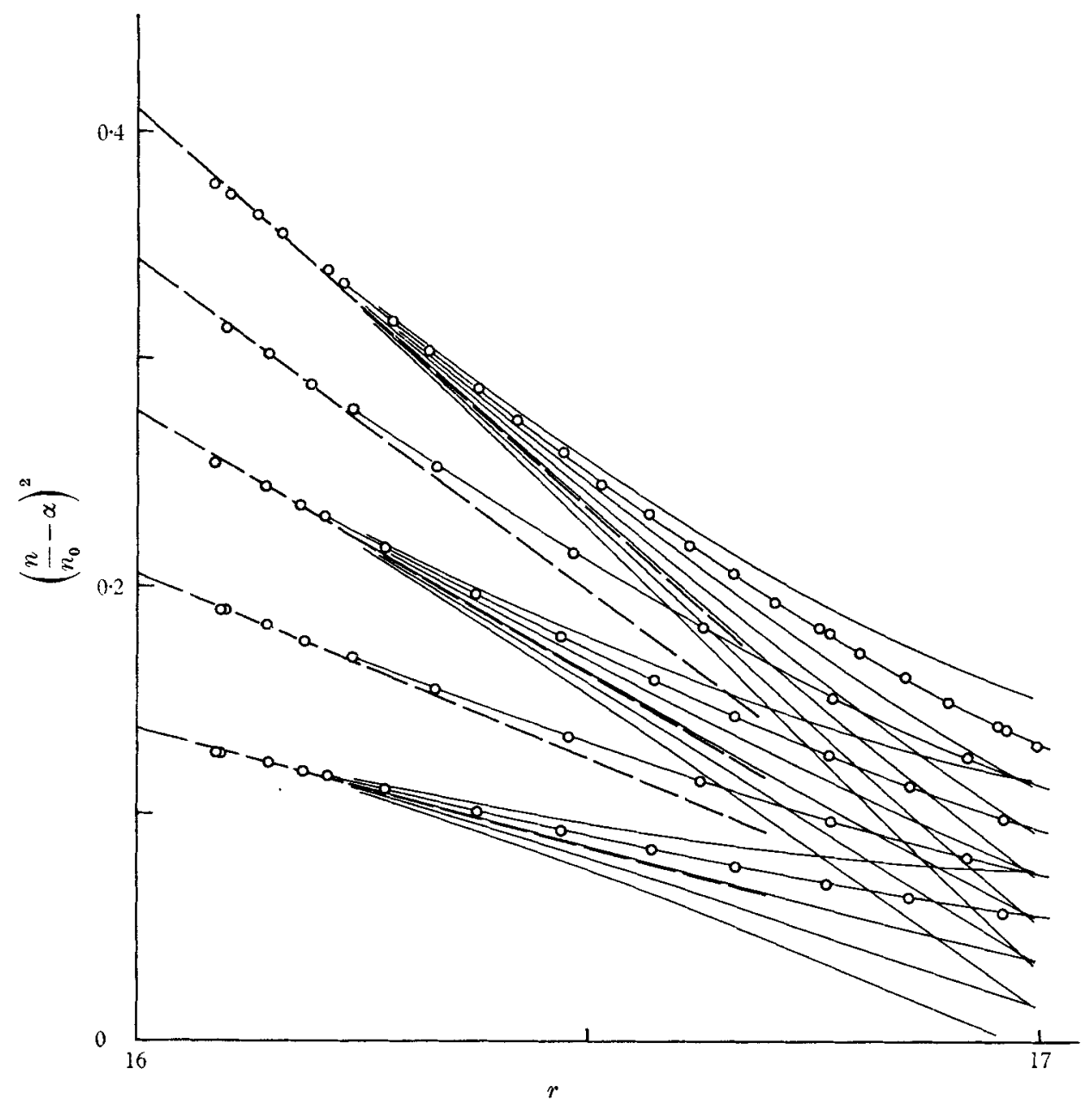

Figure $1(b)$

Figure 1. Method of extrapolation of hot-wire signals to the wall. Probe is traversed radially for several fixed angular velocities of outer cylinder. Ordinate is electrical quantity expected to be proportional to local relative velocity. (a) Data for wire 2 with probe on inner cylinder at zero pitch, zero yaw. (b) Data for wire 1 with probe on outer cylinder at zero pitch, $+15^{\circ}$ yaw.

In figure 4 , the velocity data of figure 3 for three values of cylinder speed or Reynolds number have been converted to normalized angular velocity in laboratory co-ordinates. In the region where there is an overlap in the data observed from the two cylinders, near mid-radius, the agreement is excellent. This agreement is no doubt partly due to the favourable dynamic conditions of the experiment, which were such that a probe wake was always swept radially towards the base of the probe. Thus the excess of velocity in the wake of a probe on the moving outer cylinder caused a flow deflexion outward, while the defect of velocity in the wake of a probe on the stationary inner cylinder caused a flow deflexion inward. In the pressure field of the undisturbed velocity profile, the 
initial radial acceleration of the wake fluid was of the order of $0 \cdot 2$ gravity. Under such an acceleration the wake fluid would approach the probe wall in a fraction of a revolution of the outer cylinder.

Additional evidence on this point was obtained with the aid of the third hot wire, which was mounted $180^{\circ}$ away from the data probe and was kept at a fixed radius midway between the two cylinders. For a typical rotation period of $1.545 \mathrm{sec}$ for the outer cylinder, the relative velocity at such a wire on the inner (outer) cylinder was about $3.9(1 \cdot 8) \mathrm{ft}$./sec. Figure 5 shows, in normalized form, the observed change in this velocity as the movable probe, on the same cylinder

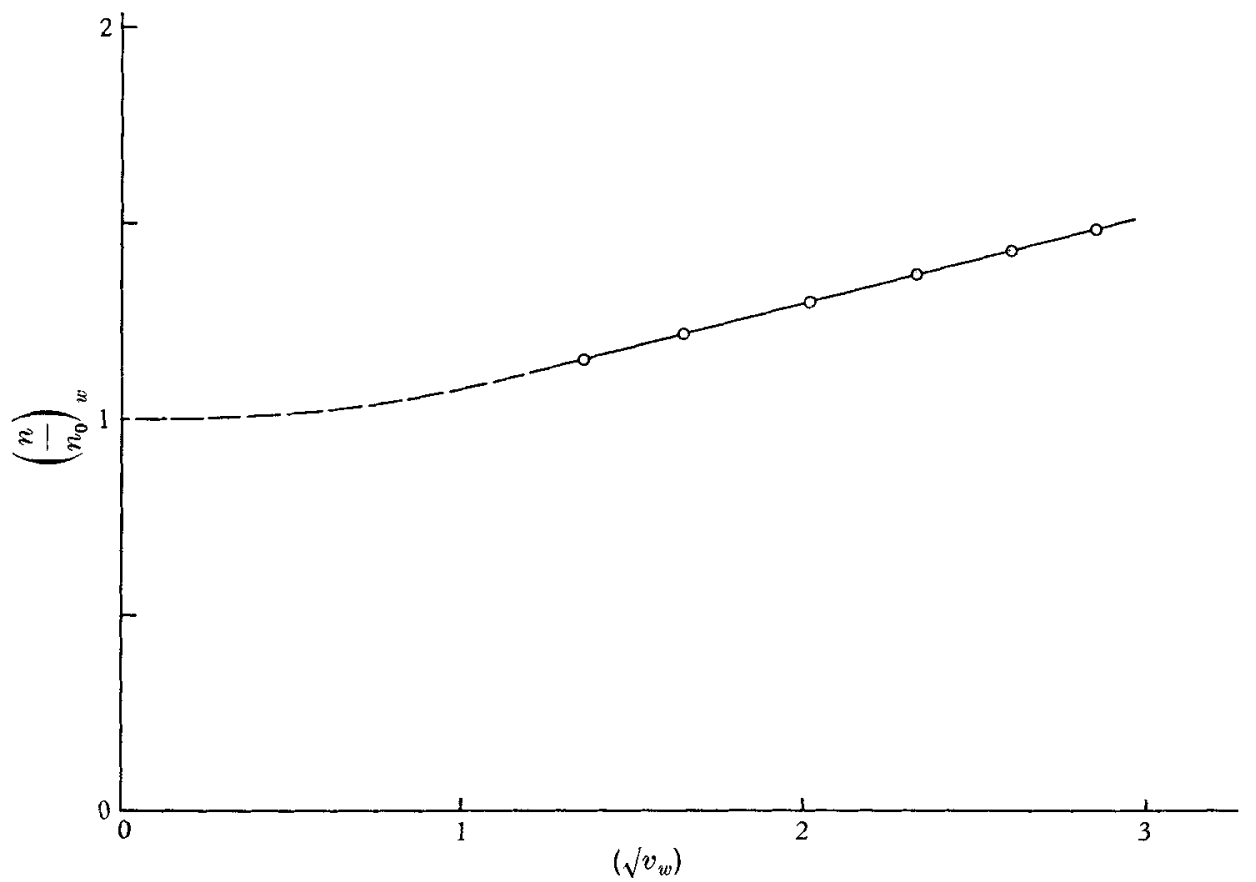

FIguRE 2. Typical hot-wire calibration. Data are for wire 1 with probe on outer cylinder at zero pitch, $+15^{\circ}$ yaw (cf. figure $\mathbf{l}(b)$ ). Ordinate is Nusselt number, normalized with freeconvection value before extrapolation to wall. Abscissa is square root of relative velocity (in ft./see) evaluated at surface of inner oylinder.

but on the far side of the annulus, was traversed from mid-radius toward the opposite wall. The maximum change is about $1 \%$, and it can be argued (on the ground that the drag of the movable probe may have varied roughly as the cube of the exposed length of stem) that the main data probe, when at mid-radius, altered the local velocity by, at most, a fraction of $1 \%$.

It is instructive to compare the real flow studied here with the theoretical flow, which is to say the steady solution of the Navier-Stokes equations for purely circulatory motion. This solution is incorporated in figure 4 as the dot-dashed line. The right-hand boundary in the figure, at $\omega / \omega_{0}=1$, represents the motion of the end plates rotating with the outer cylinder. The flow at the plane of symmetry of the apparatus is seen to be strongly distorted in the direction of the solid-body rotation imposed at the ends, the more so as the speed of rotation 


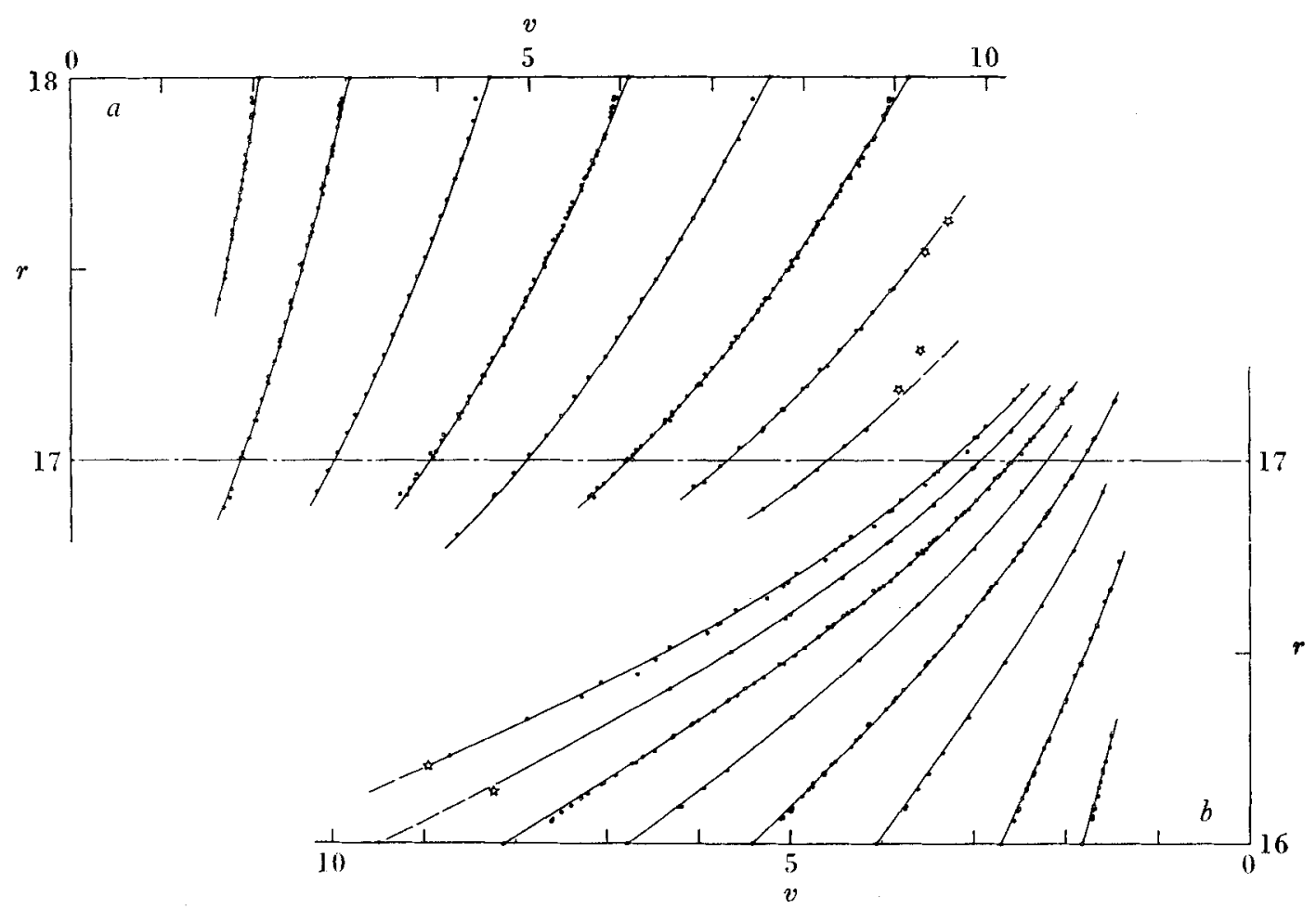

Figure 3. Relative velocity $v$ in $\mathrm{ft}$./sec between probe and fluid as observed from either cylinder for various fixed angular velocities of outer cylinder. (a) Data for probe on inner cylinder. (b) Data for probe on outer cylinder. Values shown are averages for wires 1 and 2. Probe orientation varies but has no detectable effect on flow. Stars indicate conditions at which transition to intermittently turbulent flow occurs due to instability of probe wake.

increases. One useful measure of this distortion is the ratio of the moment per unit length applied to the fluid at the two cylindrical walls, plotted as $-M_{0} / M_{i}$ in figure 6 . Because the flow is steady, the net moment must be balanced by a transport of fluid toward the plane of symmetry near the outer cylinder and away from the plane of symmetry near the inner cylinder. The sense of the secondary motion can also be argued by considering radial accelerations near the ends of the apparatus, where most of the energy dissipated in the fluid must be delivered through a thin boundary layer on the end walls.

The magnitude of the radial velocity near the plane of symmetry can be estimated from the tangential momentum equation for steady flow with circular symmetry,

$$
u \frac{\partial v}{\partial r}+\frac{u v}{r}+w \frac{\partial v}{\partial z}=v\left(\frac{\partial^{2} v}{\partial r^{2}}+\frac{1}{r} \frac{\partial v}{\partial r}-\frac{v}{r^{2}}+\frac{\partial^{2} v}{\partial z^{2}}\right)
$$

At the plane of symmetry, $w=0$. The curvature of the tangential profile in a radial view, $\partial^{2} v / \partial z^{2}$, can also be neglected compared with $\partial^{2} v / \partial r^{2}$. The last equation then amounts to an algebraic equation for the radial velocity $u(r)$ in terms of the measured tangential velocity $v(r)$. For the conditions of the present experiment, in which the profile $v / v_{0}$ as a function of $\left(r-r_{i}\right) /\left(r_{0}-r_{i}\right)$ has slope and curvature 


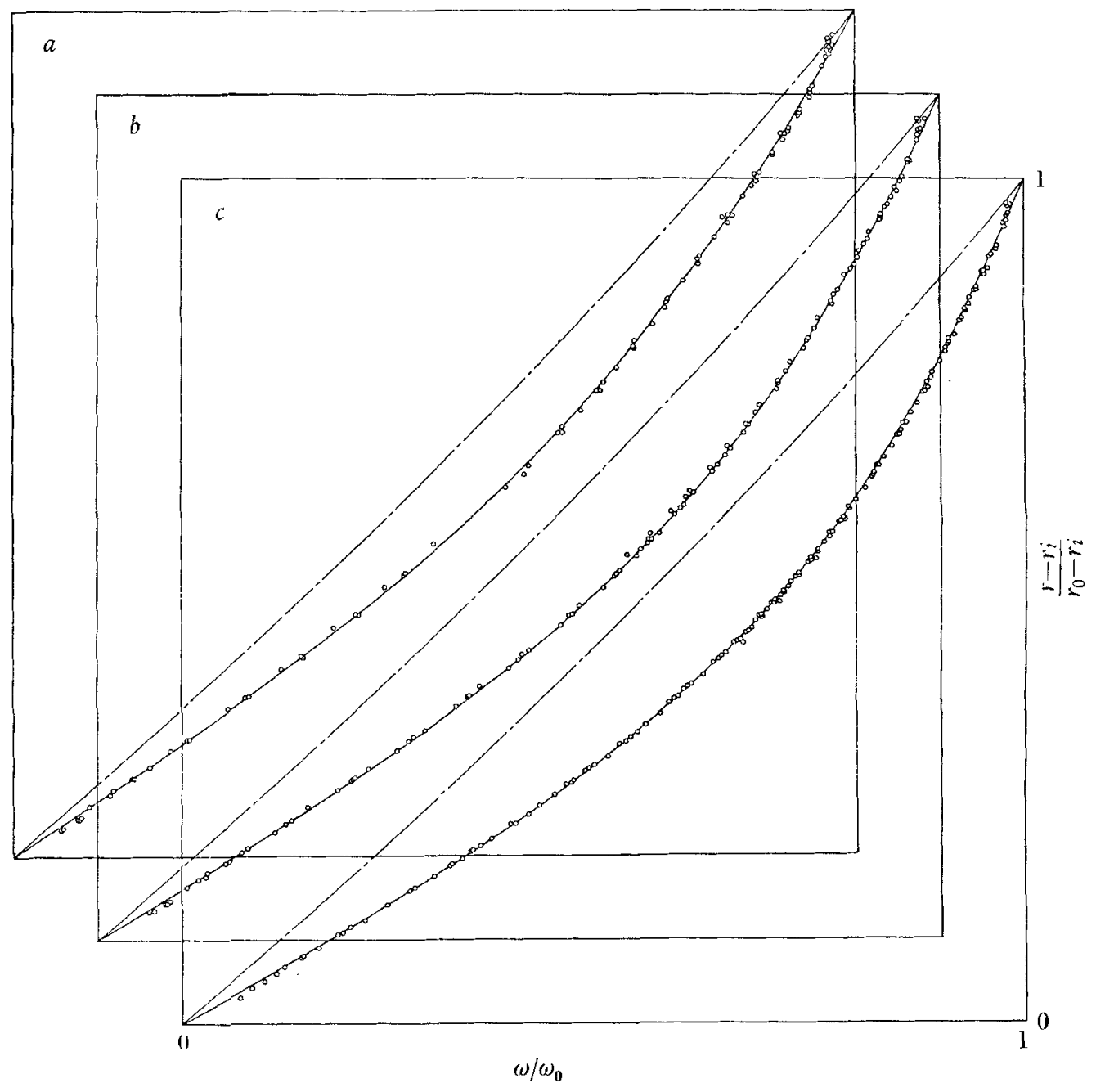

FIgure 4. Combined data showing normalized angular velocity in laboratory co-ordinates. Probe orientation varies but has no detectable effect on flow. Data in figures $4(a), 4(b)$, $4(c)$ are for $R e=\omega_{0} r_{0}\left(r_{0}-r_{i}\right) / \nu=3000,6000,9000$ respectively. Dot-dashed line shows solution of Naviex-Stokes equation for steady circulatory flow between cylinders of infinite length.

both of order unity, the normalized radial velocity $u / v_{0}$ is seen to be of order $R e^{-1}$, where $R e=v_{0}\left(r_{0}-r_{i}\right) / \nu$ is the Reynolds number based on gap and on surface velocity of the outer cylinder. This Reynolds number lies in the range 2000 12,000 , so that radial velocities near the plane of symmetry must be utterly negligible. A similar conclusion for axial velocities follows from the continuity equation.

In summary, the original object of providing a known flow for calibration purposes has evidently been achieved by the experiment. Within practical experimental accuracy, the flow in question is purely tangential in a neighbourhood of the plane of symmetry, and has the profile given in figure 4. As a 
by-product of the investigation, and as an illustration of the quasi-rigidity which is known to be typical of rotating fluids, it has also been found that end constraints in a finite laminar Couette flow at moderately high Reynolds numbers can profoundly influence the whole motion.

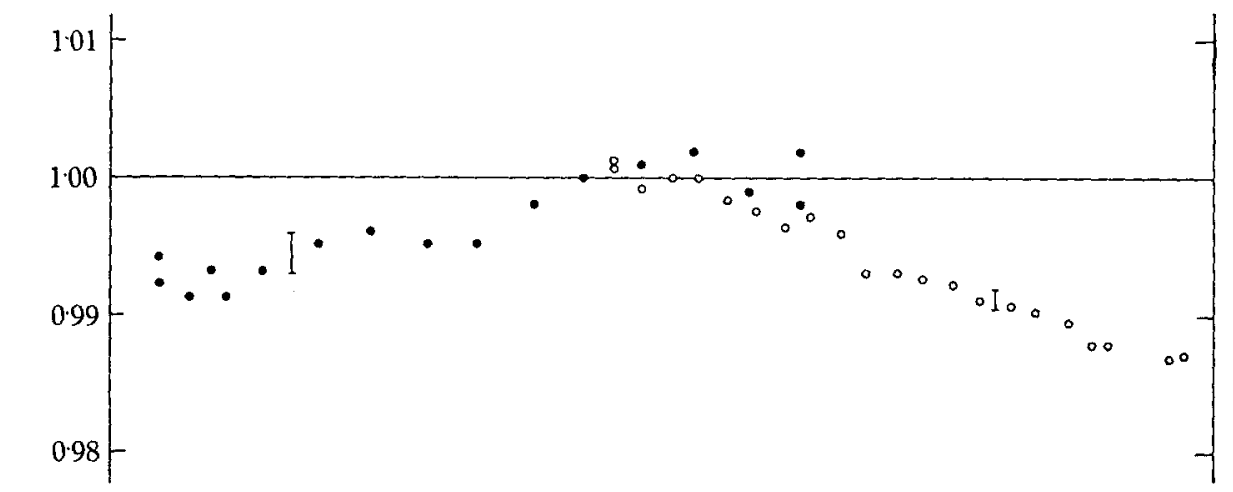

Inner cylinder

Outer cylinder

Figure 5. Effect of wake of movable probe on velocity at mid-radius on opposite side of annulus. Abscissa is radial position of tip of movable probe, mounted on outer cylinder (solid points) or on inner cylinder (open points). Ordinate is velocity indicated by fixed probe, normalized to unity when movable probe is at mid-radius. Vertical bars show resolution of digital voltmeter.

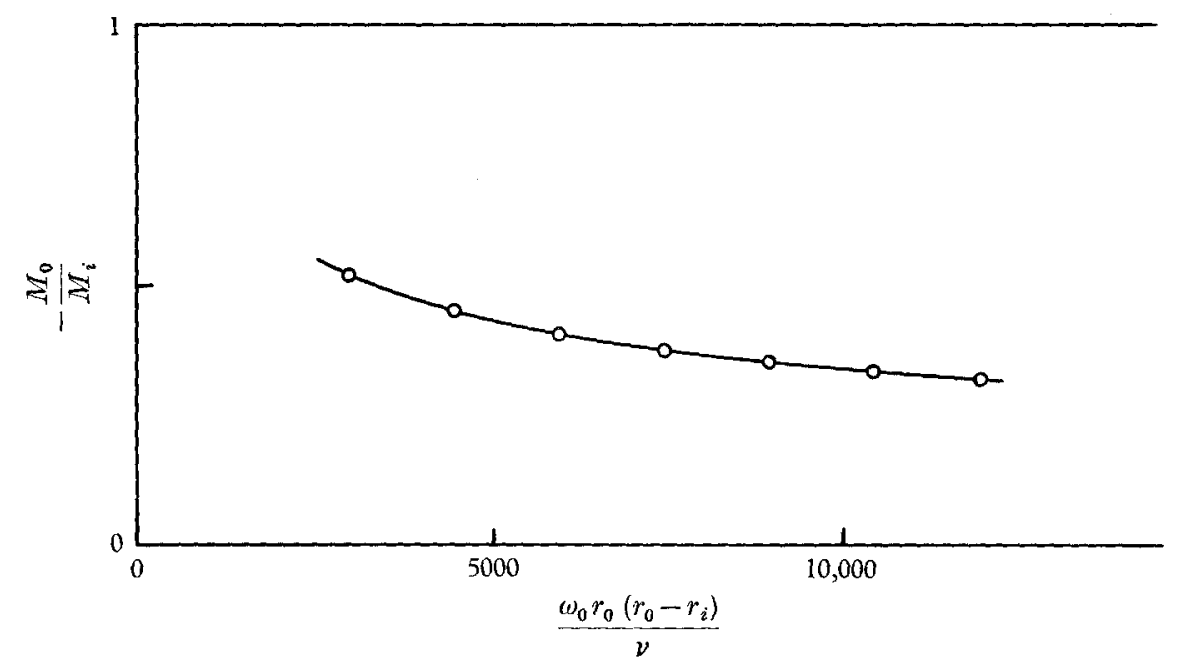

FIguRe 6. Experimentally determined ratio of moments (per unit length) applied to fluid at axial plane of symmetry by outer and inner cylinders. Ratio should be unity for purely circulatory flow.

This paper presents the results of one phase of research carried out at the Jet Propulsion Laboratory, California Institute of Technology, under Contract No. NAS 7-100, sponsored by the National Aeronautics and Space Administration.

\section{REFERENCE}

Van Atta, C. 1966 J. Fluid Mech. 25, 495. 